Aus der ${ }^{1}$ Szent Istvân Universitat, Fakultat for Agrarwissensehaften, Godölb; dem ${ }^{2}$ Institut fur Tierzucht und Tieremahrung, Herecghalom und der' Szent istván Universitat, Fakultat fur Veterinarmedizin, Budapest, Ungarn

\author{
JÁNOS PÜSKI ${ }^{1}$, SANDOR BOZÓ2, ISTVÁN GYÖRKÖS'2, ANDRÁS GÁSPẢRDY ${ }^{3}$ \\ und ENDRE SZÜCS'
}

\title{
Vergleich von vier Merkmalen innerhalb der 50-Punkte-Exterieur- beurteilung mit den erfassten Körpermaßen bei Holstein-Friesian Kïhen
}

\section{Summary}

Title of the paper: Comparison of the conformation scoring and the body measurement based on four linear traits of Holstein Friesian cows

The goal was evaluate the similarity of the body scoring system and the body measurement kept generally for the best objective method. The target-traits (withers height, body depth, rump width at the gluteal tuberosity and body capacity) of Holstein Friesian heifers were taken down. Three-three groups (-extreme, medium, textreme) based on the standard deviation of the body measurements were created and compared in each traits, as well as phenotypic relationships (regression and correlation) were calculated. Taking the relationships into consideration it appeared that the body measurements do not stand in such a close connection with each other than it is suggested by the scoring.

The pair-wise correlation coefficients between the values of the same traits taken down by scoring and measuring systems can by seen as low figures $(r=0.71,0.50,0.43$ and 0.59 , respectively) since we have the same traits. The partial correlation coefficients are even more less than the pair-wise correlation coefficients, and statistically confirmed only by the height at wither. Except the height at wither, there were no demonstrable connections in the regression of the same traits, just then, exclusively the true height at wither influenced the classifier during the scoring of all the traits.

The differences between the type groups were smaller in each traits estimated by scoring. From the analysis of the type groups it is obvious that the similarity of the two methods is the biggest in the height at wither. In the case of body depth, rump width and body capacity the classifier can distinguish only the "very little" and "very large" animals from the judged population while the intermediate types remained unrecognised. The average value of the individual identity between the two typing method was around $35 \%$.

Key Words: dairy cow, body measurements, conformation scoring, milk type groups

\section{Zusammenfassung}

Zielsetzung der Arbeit war ein Vergleich der 50-Punkte-Exterieurbeurteilung mit den gemessenen Körpermaßen. Vier Merkmale (Widerristhöhe, Rumpftiefe, Sitzbeinbreite, Körperkapazital) wurden beurteilt bzw. gemessen. Eigenheiten sowie Gleichheit der Beurteilungspunkte und der Messwerte wurden durch Gruppenbildung und Regressions- und Korrelationsrechnungen bewertet.

Bei den Beurteilungspunkten konnte ein signifikanter Unterschied zwischen den Gruppen nur bei der Widerristhöhe festgestellt werden. Die Regressions- und Korrelationsrechnungen zeigten, dasss die Zusammenhảnge zwischen den Körpermaßen in der Wirklichkeit weniger eng sind als die Exterieurbeurteilung es zeigt. Der Zusammenhang beider Bewertungsformen der Einzelmerkmale konnte bei der Widerristhöhe, der Rumpftiefe, der Sitzbeinbreite und der Körperkapazităt als nicht eng ermittelt werden $(r=0,71-0,50-0,43$ und 0,59$)$. Aufgrund der partiellen Regressionskoeffizienten konnte zwischen den gleichen Merkmalen - mit Ausnahme der Widerristhöhe - keine Regression ausgewiesen werden, Gleichzeitig konnte festgestellt werden, dass der Beurteiler bei der Schătzung aller vier Merkmale praktisch nur von den tatsăchlichen Widerristhöhen der Tiere beeinflusst wurde. Die Analyse der Typusgruppen zeigte, dass die zwei Methoden bei der Widerristhöhe am besten ibereinstimmten. Der Beurteiler konnte bezllglich Rumpftiefe, Sitzbeinbreite und Korperkapazitåt die „großen" und „kleinen" Tiere unterscheiden, die dazwischen liegenden Typen nicht. Die mit den zwei Methoden gebildeten Typusgruppen waren durchschnittlich zu $35 \%$ identisch. 
PUSKI u.a.; Vergleich von vier Merkmalen innerhalb der 50-Punkte-Exterieurbeurteilung bei Holstein-Friesian Kühen

Einleitung

Die Tierbeurteilung war in der Tierzucht lange Zeit, neben der Erfassung der Milchleistung, das wichtigste Verfahren zur Findung züchterischer Entscheidungen bei Milchkühen und spielt auch in der heutigen Tierzucht wieder eine wichtige Rolle (BREM, 1998). Die Zuchtbuchführung umfaßt die Abstammungs- und Verwandschaftsdaten, die Produktionsdaten und die Exterieurmerkmale. Beim Milchrind gehören zu den letzteren unter anderem Farben, Abzeichen, Körpermaße, Euter und bestimmte Standardmängel des Exterieurs. Das System der Exterieurbeurteilung wurde seit Anfang des vorigen Jahrhunderts ständig weiter entwickelt. Eine sogenannte „lineare Bewertung" wurde 1977 erstmals durch die nationale Vereinigung für künstliche Besamung in den USA genutzt (TRIMMBERGER et al., 1987). Im Jahre I999 wurde das Beurteilungssystem der EU, die lineare 9-Punkte-Exterieurbeurteilung auch in Ungarn eingeführt. Der Vorgänger dieses Systems war in Ungarn die auf USA-Erfahrungen basierende 50-Punkte-Methode. Wichtiger Vorteil der linearen Exterieurbeurteilung ist, dass deren Ergebnisse mit der BLUP-Methode verarbeitet werden können. Inzwischen werden in vielen Ländern diese auf den gleichen Prinzipien beruhenden Methoden angewendet, die allerdings mit verschiedenen Punkteskalen arbeiten. Die Beurteilung des Exterieurs erfolgt durch die Bewertung einzelner Exterieurmerkmale. Sie werden bewertet (geschätzt) oder gemessen. Heutzutage wird allerdings auf das Messen und auch auf das Wiegen der Tiere oft verzichtet. Anstatt exakt zu messen, werden die Körpermaße im Rahmen der Exterieurbeurteilung neben der Schätzung anderer Eigenschaften (z.B. Milchtyp, Gesamteindruck) nur bewertet. Selektions- und Züchtungsarbeit bezüglich des Exterieurs kann somit nur auf Grund vergebener Beurteilungspunkte erfolgen.

Interessanterweise trifft man - trotz umfangreicher Fachliteratur - selten auf Mitteilungen, die sich mit der Zuverlässigkeit der auf Punktesystemen basierenden Exterieurbeurteilung befassen. Aus der Sicht der Züchtungsarbeit scheint es durchaus notwendig, die in der Praxis fast ausschließlich angewendeten Punktesysteme mit der traditionellen und meist exakten Messung der Körpermaße zu vergleichen.

Das Ziel vorliegender Arbeit ist ein Vergleich der Exterieurbeurteilungspunkte mit den exakt gemessenen Körpermaßen. Die Untersuchung diente außerdem auch der Analyse yon Milchrindtypusgruppen.

Die Untersuchungen sollten zeigen, dass die Einführung des neuen 9-Punkte-Beurteilungssystems auch wegen bestehender Mängel des 50-Punkte-Beurteilungssystems notwendig ist. Die Ergebnisse sollen zur besseren Anwendung des 9-Punkte-Beurteilungssystems beitragen und gleichzeitig auf die Notwendigkeit einer objektiveren Bestimmung der Körperkapazität des Milchrindes aufmerksam machen,

\section{Material und Methoden}

Im Rahmen der Untersuchung wurden die Daten yon 159 Holstein-Friesian Kühen in der ersten Laktation bewertet. Der untersuchte Bestand stammte aus zwei Betrieben, aus der „Fríz-tej“ Genossenschaft, Kondoros $(\mathrm{n}=84)$ und aus der „Egyetértés“ Rinderviehzucht GmbH, Békés ( $n=75)$. Die durchschnittliche Milchleistung der untersuchten Kühe betrug $6150 \pm 986 \mathrm{~kg}$ (1. Laktation), das Erstkalbealter 27,2 $\pm 0,33$ Monate. Die Kühe wurden in beiden Betrieben in Tiefstreu-Laufställen gehalten, nach 
Produktionsniveau in Gruppen eingeteilt und der Milchleistung entsprechend monodiätisch gefuttert.

Die Körpermaße der Kühe wurden durch routinemässige lineare Exterieurbeurteilung und durch traditionelle Messungen bestimmt. Die Daten wurden im 2. Halbjahr 1998 erfaßt. Die Messung der Körpermaße und die Exterieurbeurteilung fand zeitgleich innerhalb der ersten 90 Tage der Laktation statt. Die Exterieurbeurteilung führte stets die gleiche Person, der offizielle Exterieurbeurteiler des Holstein-Friesian-Zuchtvereins Ungarn, durch. Die Exterieurbeurteilung der Holstein-Friesian Kühen erfolgte nach BODROGI et al. (1994). Die funktionelle Exterieurbeurteilung des ungarischen Holstein-Friesian-Bestandes ist am Anfang der 70er Jahre mit dem US-System begonnen worden. Zur Einführung der Methode wurden amerikanische Experten mit einbezogen. Das sogenannte 50-Punkte-System wurde bis zum 31. Dezember 1998 angewendet. Die Exterieurmerkmale wurden mit 1 bis 50 Punkten gewertet, wobei 50 Punkte den besten Wert darstellten. Gewertet wurden Widerristhöhe, Stärke (Brustbreite), Rumpftiefe, Hinterbeinstellung, Klauen, Fesseln, Vordereuteraufhängung, Zentralband, Eutertiefe, Hintereuterhöhe, Hintereuterbreite, Milchcharakter, Beckenneigung, Sitzbeinbreite, Hinterbeinwinkelung, Strichplazierung.

Die Hauptmerkmale werden auf einer 50-90-Skala beurteilt, hierbei werden die folgenden Merkmale gewichtet berücksichtigt; Gesamteindruck (30\%), Milchtyp (20\%), Körperkapazität $(20 \%)$, Rumpf $(30 \%)$. Der besseren Interpretierbarkeit der Punkte dient die folgende Klassifizierung:

über 90 ausgezeichnet

$85-90$

hervorragend

$80-84$

sehr gut

$75-79$

gut

$65-74$

akzeptierbar

unter 64

schlecht

Am 1. Januar 1999 wurde auf Empfehlung von ICAR auch in Ungarn, entsprechend der neuen internationalen Praxis, die 9-Punkte-Skala eingeführt,

Im Rahmen der Untersuchung wurden vier Körpermaße bzw. Merkmale (Widerristhöhe, Rumpftiefe, Sitzbeinbreite und Körperkapazität) verglichen. Die Daten wurden sowohl durch Exterieurbeurteilung als auch durch Messung aufgenommen. Bei der Widerristhöhe entspricht ein Schätzwert von 25 Punkten $142 \mathrm{~cm}$. Ein Unterschied von zwei Skalapunkten entspricht einer Differenz von einem $\mathrm{cm}$ Widerristhöhe. Als Rumpftiefe wurde der senkrechte Abstand zwischen den ausseren Punkten des Rückgrates und des Bauchs, als Sitzbeinbreite der waagerechte Abstand zwischen den äusseren Punkten der beiden Sitzbeinhőcker betrachtet. Die Korperkapazitat bedeutet das geschätzte Körpervolumen der Kühe. Die Körpermaße wurden bei der Beurteilung in Punkten, bei der Messung in $\mathrm{cm}$ ausgedrückt. Die Körperkapazität wurde einerseits im Rahmen der Exterieurbeurteilung nach Punkten bewertet, andererseits aus gemessenen Körpermaßen (Rumpflänge, Rippenbrustbreite, Beckenbodenbreite und Rumpftiefe) mit einer kleineren Änderung (anstelle der Brusttiefe Rumpftiefe) - nach LEUTHOLD et al. (1987) und SCHWARK et al. (1989) als Körpervolumenindex mit der folgenden Formel berechnet: 
Körpervolumenindex $=\frac{\frac{\text { Rippenbrustbreite }+ \text { Beckenbodenbreite }}{2} * \text { Rumpflänge } * \text { Rumpftiefe }}{1000}$

Berechnet wurden statistische Parameter wie Mittelwert, Standardabweichung und CV. Bezüglich der Körpermaße wurden 3-3er Gruppen gebildet. Basis der Gruppenbildung war die Standardabweichung der gemessenen Daten:

$$
\begin{array}{ll}
\text { Gruppe 1: } & x_{i}<\left(\bar{x}-0,5^{*} s\right) \\
\text { Gruppe 2: } & \left.(\bar{x}-0,5 * s)<x_{i}<\bar{x}+0,5^{*} s\right) \\
\text { Gruppe 3: } & x_{i}>\left(\bar{x}+0,5^{*} s\right)
\end{array}
$$

wobei $x_{i}$ den aufgenommenen Wert, $\bar{x}$ den Mittelwert und s die Standardabweichung des Merkmals darstellen.

Um den Zusammenhang zwischen gemessenen und beurteilten Körpermaßen zu prüfen, wurde die lineare Regressions- und Korrelationsanalyse durchgeführt.

Die Typusgruppen wurden nach ZELFEL (1984) gebildet. Die Kühe wurden aufgrund der gemessenen Werte von zwei Merkmalen (Widerristhöhe und Sitzbeinbreite) in folgende Typusgruppen eingeteilt: „,klein" - „schmal" (1), „klein" - ,breit" (2) „mittel" - ,schmal" (3), „,mittel" - "breit" (4), „gross" - ,schmal" (5) sowie „gross" - ,breit" (6). Durch die Bildung der Typusgruppen wurden die Unterschiede in den Körperkapazitäten dargestellt und mit den Ergebnissen der Exterieurbeurteilung verglichen.

Um einen weiteren Vergleich der zwei Methoden vornehmen zu können, wurde die Einteilung in die Typusgruppen mit beiden Erfassungsmethoden durchgefürt. Als Übereinstimmung galt, wenn das gleiche Individuum mit beiden Methoden in die gleiche Typusgruppe eingeteilt wurde. Das Ergebnis wird in Prozent mitgeteilt.

\section{Ergebnisse und Diskussion}

Die gemessenen und beurteilten Körpermaße werden in den Tabellen 1 bis 4 dargestellt. Die gemessenen Werte dienten als Basis der Gruppenbildung,

\begin{tabular}{|c|c|c|c|c|c|c|c|c|c|}
\hline \multirow[b]{2}{*}{ Gruppe (1) } & \multirow[b]{2}{*}{$\mathrm{n}$} & \multicolumn{4}{|c|}{ gemessene Widerristhøhe (2) } & \multicolumn{4}{|c|}{ beurteilte Widerristhohe (3) } \\
\hline & & $\mathrm{cm}$ & $\pm s(4)$ & $\mathrm{CV}$ & Diff. \% & Punkte (5) & $\pm \mathrm{s}(4)$ & Cy & Diff, $\%$ \\
\hline 1. „klein“ (6) & 57 & 134,6 & 1,73 & 1,3 & $-2,5$ & 13,9 & 5,0 & 36,0 & $-20,1$ \\
\hline 2. "mittel ${ }^{4}(7)$ & 54 & 138,0 & 0,87 & 0,6 & 0 & 17,4 & 4,3 & 24,7 & 0 \\
\hline 3. ...gross" (8) & 48 & 142,0 & 1,56 & 1,0 & $+2,9$ & 20,4 & 5,1 & 25,0 & $+17,2$ \\
\hline ganzer Bestand (9) & 159 & 138,0 & 3,35 & 2,4 & & 17,0 & 5,5 & 32,4 & \\
\hline $\mathrm{F}_{3 \%}$ & & 57,6 & & & & 24,1 & & & \\
\hline S.D. $.5 \%{ }^{*}(10)$ & & 0,6 & & & 0,4 & 1,9 & & & 10,8 \\
\hline S.D. $1{ }^{+}(10)$ & & 0,8 & & & 0,5 & 2,5 & & & 14,4 \\
\hline
\end{tabular}

Tabelle 1

Gemessene und beurteilte Werte der Widerristhobe (Values of the height at wither) 
Wie Tabelle 1 zeigt, betrug der Mittelwert der Widerristhöhe bei der Messung $138,0 \mathrm{~cm}$ und bei der Exterieurbeurteilung 17,0 Punkte. Ein Beurteilungswert von 17,0 Punkten entspricht nach der Umrechnungsmethode (vgl. Material und Methoden) einer Widerristhöhe von $138,0 \mathrm{~cm}$. Bei der Widerristhöhe brachten also beide Methoden das gleiche Ergebnis in Bezug auf den Mittelwert des Gesamtbestandes. Allerdings wurden die Widerristhöhen bei der Gruppe der „kleinen“ Kühe über-, bei der Gruppe der „grossen" Kühe unterschätzt. Bei der „kleinen" Gruppe betrug der Meßwert $134,2 \mathrm{~cm}$ und der Schätzwert 13,9 Punkte, bei der „grossen“ dagegen 142,0 cm bzw. 20,4 Punkte. Die aufgrund der Meßwerte berechneten Erwartungswerte der Exterieurbeurteilung betrugen bei der kleinen Gruppe 10,2 Punkte, bei der grossen 25,0 Punkte.

Die Standardabweichungen und die Variationskoeffizienten der gemessenen Werte waren sowohl im Gesamtbestand als auch in den Gruppen relativ klein. Die Standardabweichungen der Beurteilungswerte waren dagegen um eine Größenordnung höher als die der gemessenen Werte. Der Unterschied wird offensichtlich durch die verschiedenen Skalen der zwei Systeme verursacht. Aufgrund des oben Gesagten könnte die Varianz der Punkte sogar noch größer sein. Die Tatsache aber, dass der Variationskoeffizient der Punkte innerhalb der Gruppen mit dem für den Gesamtbestand festgestellten Wert als identisch betrachtet werden kann, weist auf die geringere Genauigkeit der Exterieurbeurteilung hin. Die Gruppen unterscheiden sich voneinander allerdings bei beiden Methoden signifikant.

Tabelle 2

Gemessene und beurteilte Werte der Rumpftiefe (Values of the body depth)

\begin{tabular}{|c|c|c|c|c|c|c|c|c|c|}
\hline \multirow[b]{2}{*}{ Gruppe (1) } & \multirow[b]{2}{*}{$\mathrm{n}$} & \multicolumn{4}{|c|}{ gemessene Rumpftiefe (2) } & \multicolumn{4}{|c|}{ beurteilte Rumpflicfe (3) } \\
\hline & & $\mathrm{cm}$ & $\pm s(4)$ & $\mathrm{CV}$ & Diff. $\%$ & Punkte (5) & $\pm s(4)$ & $\mathrm{CV}$ & Diff. \% \\
\hline 1. „flach" (6) & 49 & 76,1 & 1,54 & 2,0 & $-5,1$ & 17,3 & 4,8 & 27,7 & $-8,9$ \\
\hline 2. „mittel“ (7) & 60 & 80,2 & 1,15 & 1,4 & 0 & 19,0 & 3,6 & 18,9 & 0 \\
\hline 3. „ticf" (8) & 50 & 84,5 & 1,67 & 2,0 & $+5,4$ & 21,3 & 4,4 & 20,6 & $+12,1$ \\
\hline ganzer Bestand (9) & 159 & 80,3 & 3,60 & 4,5 & & 19,2 & 4,5 & 23,4 & \\
\hline $\mathrm{F}_{3 \%}$ & & 408,4 & & & & 11,4 & & & \\
\hline S.D.s\%' (10) & & 0,6 & & & 0,7 & 1,7 & & & 8,8 \\
\hline S.D. $196^{\prime}(10)$ & & 0,8 & & & 0,9 & 2,2 & & & 11,7 \\
\hline
\end{tabular}

+ kleinste signifikante Differenz

(1) Group of cows according to the average and \pm standard deviation of the measured trait, (2) Measured depth of body, (3) Scored depth of body, (4) Standard deviation، (5) Score, (6) Shallow, (7) Medium, (8) Deep, (9) Population averagc, (10) Least significant difference

Tabelle 2 zeigt tendenziöse Unterschiede zwischen den Gruppen in Bezug auf die Mittelwerte der gemessenen und beurteilten Rumpftiefe-Werte. Die Standardabweichung und der Variationskoeffizient der Rumpftiefe waren bei den gemessenen Werten wesentlich kleiner als bei den beurteilten. Die prozentualen Unterschiede zwischen den Gruppen bei den beurteilten Rumpftiefe-Werten waren kleiner als die Unterschiede bei den beurteilten Widerristhöhen, deshalb waren die Unterschiede zwischen 
den Gruppen der Tiere mit flachem und mit mittlerem Bauchprofil weniger sicher.

Die Mittelwerte der gemessenen und die beurteilten Werte der Sitzbeinbreite (Tab. 3) zeigten wiederum die erwarteten Unterschiede zwischen den Gruppen. Obwohl die gemessenen und die beurteilten Werte praktisch übereinstimmten, waren Standardabweichung und Variationskoeffizient bei den beurteilten Werten überraschend grösser. Auch dies weist auf die geringere Genauigkeit der Exterieurbeurteilung hin. Es gibt zwar einen tendenziellen Unterschied zwischen den Mittelwerten der aufgrund der beurteilten Werte gebildeten Gruppen, trotzdem finden sich in den einzelnen Gruppen "gruppenfremde" Tiere mit schmalerer oder breiterer Sitzbeinbreite. Es muss auch darauf hingewiesen werden, dass nicht einmal der erwähnte Unterschied zwischen den Gruppen erfaßbar ist, weil der Beurteiler die Kohe mit breiter Sitzbeinbreite nicht richtig erkennt.

Tabelle 3

Gemessene und beurteille Werte der Sitzbeinbreite (Values of the rump width- $3^{\text {rd }}$ (at the gluteal tuberosity))

\begin{tabular}{|c|c|c|c|c|c|c|c|c|c|}
\hline \multirow[b]{2}{*}{ Gruppe (1) } & \multirow[b]{2}{*}{$\mathrm{n}$} & \multicolumn{4}{|c|}{ gemessene Sitzbeinbreite (2) } & \multicolumn{4}{|c|}{ beurteilte Sitzbeinbreite (3) } \\
\hline & & $\mathrm{cm}$ & $\pm s(4)$ & CV & Diff. \% & Punkte (5) & ts (4) & $\mathrm{CV}$ & Diff. \% \\
\hline 1. „dUnn" (6) & 42 & 19,0 & 0.72 & 3.8 & $-10,0$ & 15,6 & 4,3 & 27,6 & $-15,7$ \\
\hline 2., "mittel" (7) & 70 & 21,1 & 0,50 & 2,4 & 0 & 18,5 & 4,3 & 23,2 & 0 \\
\hline 3. „breit" (8) & 47 & 23,2 & 0,79 & 3,4 & $-9,9$ & 19,1 & 4,0 & 20,9 & $+3,2$ \\
\hline ganzer Bestand (9) & 159 & 21,2 & 1,71 & 8,1 & & 17,9 & 4,4 & 24,6 & \\
\hline$F_{s v_{6}}$ & & 463,0 & & & & 9,1 & & & \\
\hline S.D.. $\mathrm{ss}^{+}(10)$ & & 0,26 & & & 1,2 & 1.6 & & & 8,9 \\
\hline S.D.1\% ${ }^{+}(10)$ & & 0,34 & & & 1,6 & 2,2 & & & 11,8 \\
\hline
\end{tabular}

+ kleinste signifikante Differenz.

(1) Group of cows according to the average and \pm standard deviation of the measured trait, (2) Measured rump width. (3) Scored funp width, (4) Standerd deviation, (5) Score, (6) Narrow, (7) Medium, (8) Wide, (9) Population average, (10) Least significant difference

Tabelle 4

Berechnete und beurteilte Werte der Korperkapazitat (Values of the body capacity)

\begin{tabular}{|c|c|c|c|c|c|c|c|c|c|}
\hline \multirow[b]{2}{*}{ Gruppe (1) } & \multirow[b]{2}{*}{$\mathrm{n}$} & \multicolumn{4}{|c|}{ berechnete Ko̊rperkapazitát (2) } & \multicolumn{4}{|c|}{ beurteilte Körperkapazităt (3) } \\
\hline & & $\mathrm{dm}^{3}$ & $\pm s(4)$ & CV & Diff. \% & Punkte (5) & ts (4) & $\mathrm{CV}$ & Dift. \% \\
\hline I. „klein ${ }^{4}(6)$ & 53 & 490 & 28,3 & 5,8 & $-11,9$ & 70,5 & 4,0 & 5,7 & $-4,3$ \\
\hline 2., "mittel" (7) & 58 & 556 & 17,1 & 3,1 & 0 & 73,6 & 3,7 & 5,0 & 0 \\
\hline 3. "gross" (8) & 48 & 621 & 22,6 & 3,6 & $+11,6$ & 74,6 & 3,6 & 4,8 & $+1,4$ \\
\hline ganzer Bestand (9) & 159 & 554 & 57,3 & 10,3 & & 72,9 & 4,1 & 5,6 & \\
\hline$F_{s \%}$ & & 412,9 & & & & 16,5 & & & \\
\hline S.D.sw ${ }^{+}(10)$ & & 9,0 & & & 1,6 & 1,5 & & & 2,0 \\
\hline S.D. ${ }_{1 \%}{ }^{*}(10)$ & & 12,0 & & & 2,2 & 2,0 & & & 2,7 \\
\hline
\end{tabular}


Die Tabelle 4 enthält die aus den gemessenen Körpermassen berechneten und die durch Exterieurbeurteilung gewonnenen Körperkapazität-Werte. Der Variationskoeffizient war bei der aus den gemessenen Körpermassen berechneten Körperkapazität größer als bei der durch Exterieurbeurteilung ermittelten Körperkapazität. Es war also umgekehrt, als bei den bisher diskutierten Merkmalen, während die Tendenz, dass Standardabweichungen und Variationskoeffizienten der Gruppen kleiner waren als die des Gesamtbestandes, gleich blieb. Während bei der berechneten Körperkapazität die Unterschiede zwischen den Gruppen (12-12\%) statistisch gesichert waren, bestand bei der durch Exterieurbeurteilung gewonnenen Körperkapazität zwischen den ,,mittleren“ und ,grossen" Gruppen kein statistisch gesicherter Unterschied. Die Exterieurbeurteilung - ähnlich wie bei der Sitzbeinbreite - unterschätzt die Körperkapazität der großen Kühe.

\section{Tabelle 5}

Zusammenhänge zwischen den gemessenen Merkmalen ( $\mathrm{I}).(\mathrm{n}=159)$ (Relationships between the measured traits $(\mathrm{n}=159))$

\begin{tabular}{|c|c|c|c|c|c|c|}
\hline & Merkmal (1) & & $\frac{\text { I. }}{\text { Widerristhohe (2) }}$ & $\frac{\text { I. }}{\text { Rumpftiefe (3) }}$ & $\begin{array}{c}\text { I. } \\
\text { Sitzbeinbreite (4) }\end{array}$ & $\begin{array}{c}\text { I. } \\
\text { Körperkapazităt } \\
\text { (5) }\end{array}$ \\
\hline \multirow[t]{3}{*}{ I. } & Widerristhöhe (2) & $r^{+1}(6):$ & 1,00 & $0,54 * \leftrightarrow$ & $0,52 \cdots$ & $0,69 \cdots$ \\
\hline & & $r_{p}^{+2}(7):$ & - & -0.12 & 0,02 & $0,44 * 6 \%$ \\
\hline & & $b_{p}^{+3}(8):$ & - & $-0,08$ & 0,02 & $0,24 * \cdots$ \\
\hline \multirow[t]{3}{*}{ I. } & Rumpftiefe (3) & $f^{+1}(6):$ & . & 1,00 & 0,58 & $0,85 * *+$ \\
\hline & & $r_{p}+2(7):$ & + & - & $-0,11$ & 0,73 \\
\hline & & $b_{p}{ }^{+3}(8):$ & $-0,16$ & + & $-0,14$ & 0,55 a.e \\
\hline \multirow[t]{3}{*}{ I. } & Sitzbeinbreite (4) & $\mathrm{r}^{\prime 1}(6):$ & - & - & 1,00 & $0,73 \cdots$ \\
\hline & & $r_{p}^{+2}(7):$ & . & + & - & $0,49 * 4$ \\
\hline & & $b_{p}{ }^{43}(8):$ & 0,03 & $-0,08$ & - & $0,28 * * *$ \\
\hline \multirow[t]{7}{*}{ l. } & Körperkapazităt (5) & $r^{+1}(6):$ & - & - & - & 1,00 \\
\hline & & $\mathrm{r}_{\mathrm{p}}{ }^{2}(7):$ & - & - & - & - \\
\hline & & $b_{p}^{+3}(8):$ & $0,81 \bullet .6$ & $0,97 * \bullet$ & $0,83 * * \bullet$ & $-i$ \\
\hline & & $\mathrm{R}^{2 * 4}(9)=$ & 0,483 & 0,729 & 0,542 & $0,845(85 \%)$ \\
\hline & & $\begin{array}{r}\mathrm{R}^{2 * 5}(10) \\
=\end{array}$ & 0,695 & 0,853 & 0,736 & 0,919 \\
\hline & & $\mathrm{F}=$ & 48,34 & 139,11 & 61,10 & 281,42 \\
\hline & 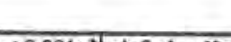 & $p<$ & 0,000 & 0,0000 & 0,0000 & 0,0000 \\
\hline
\end{tabular}

Die Übereinstimmung der gemessenen und der durch Exterieurbeurteilung geschälzten Körpemaße wurde durch die Berechnung von einfachen und partiellen Korrelationsko- 
effizienten untersucht, während die Wechselwirkungen der mit den beiden Methoden festgestellten Körpermaße mit den partiellen Regressionskoeffizienten ausgedrückt wurden. In Tabelle 5 werden die Zusammenhänge zwischen den gemessenen, in Tabelle 6 die Zusammenhänge zwischen den beurteilten Körpermaßen dargestellt aus denen die Besonderheit beider Methoden erkennbar ist.

Tabelle 6

Zusammenhănge zwischen den beurteilten Merkmalen (II.) $(\mathrm{n}=159)$ (Relationships between the scored traits ( $\mathrm{n}$ =159)

\begin{tabular}{|c|c|c|c|c|c|c|}
\hline & Merkmal (1) & & $\frac{\text { Il. }}{\text { Widerristhohe (2) }}$ & $\stackrel{\text { II. }}{\text { Rumpftiefe (3) }}$ & $\begin{array}{l}\text { II. } \\
\text { Sitzbeinbreite (4) }\end{array}$ & $\begin{array}{c}\text { II. } \\
\text { Kobrperkapazita! } \\
\text { (5) }\end{array}$ \\
\hline \multirow[t]{3}{*}{ II. } & Widerristhōhe (2) & $r^{+1}(6):$ & 1,00 & $0,85 * * *$ & $0,62 * \cdots$ & 0,86 \\
\hline & & $f_{p}{ }^{2}(7):$ & - & $0,33 \cdots$ & 0,06 & $0,35 \cdots$ \\
\hline & & $b_{p}^{+3}(8):$ & - & $0,26 * 4 *$ & 0,09 & $0,27 * \bullet$ \\
\hline \multirow[t]{3}{*}{ II. } & Rumpftiefe (3) & $r^{+1}(6):$ & - & 1,00 & $0,65 \cdots$ & $0,91 *$ \\
\hline & & $r_{p}{ }^{2}(7):$ & - & - & 0,06 & $0,64 * .4$ \\
\hline & & $b_{p}{ }^{+3}(8):$ & $0,41 \cdots$ & - & 0,12 & $0,61 * * *$ \\
\hline \multirow[t]{3}{*}{ II. } & Silzbeinbreite (4) & $r^{* 1}(6):$ & - & - & 1,00 & $0,68 * * *$ \\
\hline & & $r_{p}{ }^{\prime 2}(7):$ & - & - & $=$ & 0,25 *at \\
\hline & & $b_{p}{ }^{3}(8):$ & 0,04 & 0,03 & - & $0.12 \cdots$ \\
\hline \multirow[t]{7}{*}{ II. } & Körperkapazitat (5) & $r^{+1}(6):$ & - & - & - & 1,00 \\
\hline & & $r_{p}+2(7):$ & - & - & - & - \\
\hline & & $b_{p}{ }^{3}(8):$ & $0,46 \cdots$ & $0,67 * 0 *$ & $0,49 \cdots$ & - \\
\hline & & $\mathrm{R}^{2+4}(9)=$ & 0,769 & 0,853 & 0,467 & $0,866(87 \%)$ \\
\hline & & $\begin{array}{r}\mathrm{R}^{2+5}(10) \\
=\end{array}$ & 0,877 & 0,924 & 0,684 & 0,931 \\
\hline & & $F=$ & 172,24 & 299,58 & 45,35 & 334,92 \\
\hline & & $\mathrm{p}<$ & 0,000 & 0,0000 & 0,0000 & 0,0000 \\
\hline
\end{tabular}

"6* $p<0,001 ;{ }^{+1}$ einfacher Korrelationskoeffizient; ${ }^{+2}$ partieller Kontelationskoeffizient; ${ }^{-3}$ partieller Regressionskoeffizient;

${ }^{4}$ Bestimmtheitsmass; " mehrfacher Korrelationskoeffizient

(1) Traih, (2) Scored height at wither, (3) Scored body depth, (4) Scored rump width-3" $3^{\text {rd }}$. (5) Scored body capacity, (6) Pair-wise correlation,

(7) Partial correlation, (8) Partial regression, (9) Cocfficient of determination, (10) Multiple correlation coefficient

Die einfachen Korrelationskoeffizienten zwischen Widerristhöhe und Rumpftiefe $(r=0,52)$, Widerristhöhe und Sitzbeinbreite $(r=0,54)$ sowie Rumpftiefe und Sitzbeinbreite $(r=0,58)$ zeigen mittlere Schätzwerte, während die Korrelationen der drei erwähnten Merkmale zur Körperkapazität $(r=0,69,0,73,0,85)$ wesentlich enger waren (Tab. 5). Die Korrelationen zwischen den drei Körpermaßen und der Körperkapazität waren deshalb enger, weil alle drei Merkmale Bestandteil des Körpervolumenindex 
sind. Die Beziehungen zwischen Widerristhöhe, Rumpftiefe und Sitzbeinbreite waren dagegen deshalb geringer, weil das untersuchte Tiermaterial vermutlich aus unterschiedlichen Körperbautypen bestand, also keine homogene Population darstellte. Diese Feststellung wird durch die partiellen Korrelationskoeffizienten eindeutig unterstülzt. Der Zusammenhang zwischen zwei Merkmalen (mit Ausnahme der Körperkapazität) verschwindet, sobald eine neue Variable mit einbezogen wird $\left(r_{p}=0,02\right.$, $0,11,0,12)$. Auch die partiellen Regressionskoeffizienten $\left(b_{p}=0,02\right.$ bis $\left.-0,16\right)$ zeigen, dass die Körpermaße (mit Ausnahme der Körperkapazität, wo ein kausaler Zusammenhang besteht) relativ unabhängig von einander waren,

Im Falle der Exterieurbeurteilung (Tab, 6) waren die einfachen Korrelationskoeffizienten zwischen den einzeInen Körpemaßen sowie zwischen den Körpermaßen und der Körperkapazität enger als bei den gemessen Werten $(r=0,62,0,65$ und 0,85 , bzw. $r=0,68,0,86$ und 0,91 ). Dies läßt die Vermutung $z u$, dass weniger differenzierte Angaben durch Exterieurbeurteilung erhalten werden können als durch die Messung. Die Unsicherheit der Exterieurbeurteilung gegenüber der Messung zeigte der signifikante partielle Korrelationskoeffizient zwischen der Widerristhöhe und der Rumpftiefe $\left(\mathrm{r}_{\mathrm{p}}=0,33\right)$. Die gleichfalls signifikanten partiellen Regressionskoeffizienten $\left(b_{p}=0,26\right.$ und 0,41$)$ erlauben die Aussage, dass der Beurteiler dazu neigte einer Kuh mit grosser Widerristhöhe auch eine grosse Rumpftiefe zuzuweisen, auch wenn dies nicht zutriff. Die Koeffizienten zeigten ausserdem, dass der Beurteiler bei der Abschätzung der Körperkapazität die Sitzbeinbreite nur ungenügend berücksichtigte.

In Tabelle 7 sind die Zusammenhänge zwischen den mit verschiedenen Methoden erfaßten Körpermaßen dargestellt. Die engste Beziehung wurde zwischen den gemessenen und beurteilten Werten bei der Widerristhöhe gefunden $(r=0,71)$. Dieser Zusammenhang gilt jedoch nur mit Einschränkungen, da es sich um die Widerristhöhe bei dem gleichen Tier handelt. Bei den Merkmalen Rumpftiefe, Sitzbeinbreite und Körperkapazität ergaben sich zwischen den gemessenen und beurteilten Werten mittlere Schätzwerte $(x=0,50,0,43$ und 0,59$)$. Die partiellen Korrelationskoeffizienten zeigten zwischen den gemessenen und beurteilten Werten bei allen Merkmalen einheitlich geringere Beziehungen, die nur bei der Widerristhöhe statistisch zu sichern war. Die partiellen Regressionskoeffizienten zeigten eine ähnliche Tendenz. Zwischen den gemessenen und beurteilten Werten konnte - mit Ausnahme der Widerristhöhe - keine Regression ausgewiesen werden. Es kann angenommen werden, dass der Beurteiler bei der Erfassung der Merkmale vor allem durch die tatsächliche Widerristhöhe des Tieres beeinflusst wurde. Der Beurteiler neigte also dazu, einem Tier mit großer Widerristhöhe auch eine große Rumpftiefe und Sitzbeinbreite, aber auch eine große Körperkapazität zuzuordnen. Eine Ausnahme bildete die Sitzbeinbreite, auf deren Beurteilung auch die tatsächliche Rumpftiefe Einfluß ausübte. Merkwürdigerweise konnte aber kein Einfluss der gemessenen Sitzbeinbreite auf die Beurteilung der Sitzbeinbreite nachgewiesen werden.

Um die gemessenen und die durch Exterieurbeurteilung geschätzten Werte vergleichen zu können, wurden die Tiere aufgrund der tatsächlichen Widerristhöhen und Sitzbeinbreiten in Typusgruppen eingeteilt. Die Ergebnisse dieses Vergleiches sind in Tabelle 8 zusammengefaßt.

Es ist zu bemerken, dass die einzelnen Merkmale sich nach den Bedingungen der 
Tabelle 7

Zusammenhänge zwischen gemessenen (I.) und beurteilten Merkmalen (II.) $(\mathrm{n}=159)$ (Relationships between the measured (1.) and scored traits $(\mathrm{n}=159)$ )

\begin{tabular}{|c|c|c|c|c|c|c|}
\hline & Merkmal (1) & & $\begin{array}{c}\text { II. } \\
\text { Widerristhöhe (11) }\end{array}$ & $\begin{array}{l}\text { II. } \\
\text { Rumpttiefe (12) }\end{array}$ & $\begin{array}{c}\text { II, } \\
\text { Sitzbeinbreite (13) }\end{array}$ & $\begin{array}{c}\text { II. } \\
\text { Körperkapazităt (14) }\end{array}$ \\
\hline \multirow[t]{3}{*}{ I. } & Widerristhöhe (2) & $r^{-1}(6)$ & $0,71 \approx 00$ & $0,44 * 0$ & 0,44 **t & $0,56 * 0 *$ \\
\hline & & $t_{\mathrm{p}}{ }^{+2}(7):$ & 0.52 & $0,46^{* 0.9}$ & $0,33 * * *$ & $0,50 * *$ \\
\hline & & $b_{p}{ }^{+1}(8):$ & 0,60 & $0,53 * \ldots$ & $0,39 \cdots$ & $0,56 * * *$ \\
\hline \multirow[t]{3}{*}{1.} & Rumpfliefe (3) & $r^{+1}(6):$ & $0,66 * 4 *$ & $0,50 \cdots$ & $0,41 \cdots$ & $0,57 * * *$ \\
\hline & & $r_{p}^{+2}(7):$ & $-0,03$ & 0,10 & $-0,17^{\circ}$ & $-0,05$ \\
\hline & & $b_{p}{ }^{3}(8):$ & $-0,04$ & 0,14 & $-0,27^{*}$ & $-0,06$ \\
\hline \multirow[t]{3}{*}{ 1. } & Sitzbeinbreite (4) & $r^{+1}(6):$ & $0,56 \cdots$ & 0,32 *ke & $0,43 \cdots$ & $0,49 * * 4$ \\
\hline & & $r_{p}{ }^{2}(7):$ & 0,03 & 0,06 & 0,10 & 0.06 \\
\hline & & $b_{p}^{23}(8):$ & 0,04 & 0.11 & 0,12 & 0,07 \\
\hline \multirow[t]{7}{*}{ I. } & Korperkapazitat (5) & $r^{+1}(6):$ & $0,71^{4 * *}$ & $0,46 \cdots$ & $0,48 * 4$. & $0,59 * * *$ \\
\hline & & $r_{p}^{+2}(7):$ & 0,09 & 0,06 & 0,18 * & 0,12 \\
\hline & & $b_{p}^{-3}(8):$ & 0.16 & 0,11 & $0,37 *$ & 0,22 \\
\hline & & $\mathrm{R}^{2+4}(9)=$ & 0,510 & 0,467 & 0,362 & $0.523(52 \%)$ \\
\hline & & $\mathrm{R}^{2+5}(\mathrm{I} 0)=$ & 0,714 & 0,684 & 0,602 & 0,723 \\
\hline & & $\mathrm{F}=$ & 40,01 & 33,76 & 21,84 & 42,25 \\
\hline & & $\mathrm{p}<$ & 0,000 & 0,0000 & 0,0000 & 0,0000 \\
\hline
\end{tabular}

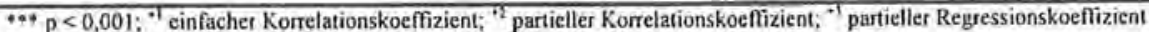

"Bestimmtheitsmass; ${ }^{* 9}$ mehrfacher Korrelationskoeffizient

(1) Trait, (2) Measured height at wither, (3) Measured body depth, (4) Measured rump width-3 $3^{\text {id }}$, (5) Calcualated body capacity (see in Table 4.), (6) Pair-wise correlation. (7) Partial correlation, (8) Partial regression, (9) Coefficient of determination, (10) Multiple correlation coefficient, (11) Scored height at wither, (12) Scored body depth, (13) Scored rump width-3 $3^{\text {rd }}$. (14) Scored body capacity,

Typusbildung und nach den Beziehungen zwischen den Merkmalen änderten. Die Werte der Widerristhöhe stiegen bei beiden Methoden ähnlich stufenweise. Für die Merkmale Rumpftiefe, Sitzbeinbreite und Körperkapazität dagegen galt dies weniger deutlich.

Bei der Exterieurbeurteilung grenzte sich bezüglich geschätzter Rumpftiefe nur die Gruppe „klein-schmal-flach“ von den anderen ab. Bezüglich beurteilter Sitzbeinbreite bildeten gleichfalls die „kleinen-schmalen" Kühe eine selbständige Gruppe. Diese Feststellungen zeigen, dass der Beurteiler eigentlich nur die erste Typusgruppe, die „kleinen" Kühe erkennen konnte, die restlichen fünf Typen wurden als ein Typ wahrgenommen. Diese strenge Gruppierung wurde durch die in der Körperkapazität erkennbare Dreiergliederung etwas aufgelockert. Die Dreiergliederung der beurteilten Körperkapazität ist immer noch nicht differenziert genug, weil sie die tatsächlichen Unterschiede bzw. Ähnlichkeiten zwischen den Typen nicht befriedigend darstellt. 


\section{Tabelle 8}

Gemessene und beurteilte Werte der Merkmale bei den verschiedenen Typusgruppen (Values of the measured and scored traits by type group)

\begin{tabular}{|c|c|c|c|c|c|c|c|c|}
\hline \multirow{5}{*}{ Merkmal (I) } & \multicolumn{8}{|c|}{ Typusgruppe (2) } \\
\hline & & \multicolumn{2}{|c|}{ klein (3) } & \multicolumn{2}{|c|}{ mittel (4) } & \multicolumn{2}{|c|}{ gross (5) } & \multirow{4}{*}{$\begin{array}{c}\mathrm{F}_{5 \%}=1 \\
\mathrm{~S} \mathrm{D}_{.5 \%}=1 \\
\mathrm{~S} \mathrm{D}_{1 \%}=1 \\
(17)\end{array}$} \\
\hline & \multirow[b]{3}{*}{$\mathrm{n}$} & dünn (6) & breit (7) & dânn (6) & breit (7) & đünn (6) & breit (7) & \\
\hline & & No. 1 & No. 2 & No. 3 & No. 4 & No. 5 & No. 6 & \\
\hline & & 28 & 29 & 32 & 22 & 25 & 23 & \\
\hline \multicolumn{9}{|l|}{ Widerristhøhe (8) } \\
\hline \multirow[t]{4}{*}{ gemessen (9) } & $\bar{x}(15)$ & 133,7 & 135,4 & 138,0 & 138,0 & 141,8 & 142,2 & $164,7 * *$ \\
\hline & $\pm s(16)$ & 1,94 & 0,90 & 0,86 & 0,92 & 1,55 & 1,58 & 0,8 \\
\hline & $\mathrm{CV}$ & 1,5 & 0,7 & 0,6 & 0,7 & 1,1 & 1,1 & 1,0 \\
\hline & $\%$ & 100 & 101 & 103 & 103 & 106 & 106 & \\
\hline \multirow[t]{4}{*}{ beurteilt $(10)$} & $\bar{x}(15)$ & 12,1 & 15,6 & 17,3 & 17,6 & 20,7 & 20,0 & $11,5 * * *$ \\
\hline & $\pm s(16)$ & 5,6 & 3,8 & 4,0 & 4,8 & 5,2 & 5,0 & 2,6 \\
\hline & $\mathrm{CV}$ & 46,3 & 24,4 & 23,1 & 27,3 & 25,1 & 25,0 & 3,6 \\
\hline & $\%$ & 100 & 128 & 143 & 145 & 171 & 165 & \\
\hline \multicolumn{9}{|l|}{ Rumpftiefe (11) } \\
\hline \multirow[t]{4}{*}{ gemessen (9) } & $\bar{x}(15)$ & 76,9 & 80,4 & 79,3 & 81,5 & 81,6 & 83,1 & $12,8 * * *$ \\
\hline & $\pm s(16)$ & 2,86 & 3,43 & 2,39 & 3,13 & 3,77 & 2,73 & 1,7 \\
\hline & $\mathrm{CV}$ & 3,7 & 4,3 & 3,0 & 3,8 & 4,6 & 3,3 & 2,3 \\
\hline & $\%$ & 100 & 105 & 10,3 & 106 & 106 & 108 & \\
\hline \multirow[t]{4}{*}{ beurteilt $(10)$} & $\bar{x}(15)$ & 15,4 & 19,0 & 18,8 & 19,9 & 21,7 & 21,0 & $7,5 \cdots$. \\
\hline & $\pm s(16)$ & 4,4 & 3,3 & 4,2 & 3,3 & 4,5 & 5,0 & 2,3 \\
\hline & $\mathrm{cV}$ & 28,6 & 17,4 & 22,3 & 16,6 & 20,7 & 23,8 & 3,1 \\
\hline & $\%$ & 100 & 123 & 122 & 129 & 141 & 136 & \\
\hline Sitzbeinbreite (12) & & & , & & & & & \\
\hline \multirow[t]{4}{*}{ gemessen (9) } & $\bar{x}(15)$ & 18,8 & 21,6 & 20,4 & 22,8 & 20,8 & 23,3 & 90,9 *t* \\
\hline & $\pm s(16)$ & 0,8 & 0,9 & 0,9 & 1,0 & 0,8 & 0,8 & 0,5 \\
\hline & $\mathrm{CV}$ & 4,3 & 4,2 & 4,4 & 2,3 & 3,8 & 3,4 & 0,7 \\
\hline & $\%$ & 100 & 115 & 109 & 121 & 111 & 124 & \\
\hline \multirow[t]{4}{*}{ beurteilt (10) } & $\bar{x}(15)$ & 15,0 & 18,4 & $\mathrm{i} 7,4$ & 17,9 & 19,2 & 20,2 & $4,8 * * *$ \\
\hline & $\pm s(16)$ & 4,0 & 3,7 & 4,5 & 4,3 & 5,0 & 3,4 & 2,3 \\
\hline & $\mathrm{CV}$ & 26,7 & 20,1 & 25,9 & 24,0 & 26,0 & 16,8 & 3,1 \\
\hline & $\%$ & 100 & 123 & 116 & 119 & 128 & 135 & \\
\hline \multicolumn{9}{|l|}{ Körperkapazitat (13) } \\
\hline \multirow[t]{4}{*}{ berechnet (13) } & $\bar{x}(15)$ & 491 & 549 & 535 & 583 & 574 & 611 & $23,7 \cdots$ \\
\hline & $\pm s(16)$ & 43 & 53 & 39 & 37 & 52 & 31 & 24 \\
\hline & $\mathrm{CV}$ & 8,8 & 9,6 & 7,3 & 6,3 & 9,1 & 5,1 & 33 \\
\hline & $\%$ & 100 & 112 & 109 & 119 & 117 & 124 & \\
\hline \multirow[t]{4}{*}{ beurteilt (10) } & $\bar{x}(15)$ & 69,1 & 72,3 & 72,6 & 73,1 & 75,5 & 75,5 & $11,7 \ldots$ \\
\hline & Is (16) & 3,9 & 2,8 & 3,8 & 3,1 & 3,4 & 4,2 & 2,0 \\
\hline & $\mathrm{CV}$ & 5,6 & 3,9 & 5,2 & 4,2 & 4,5 & 5,6 & 2,7 \\
\hline & $\%$ & 100 & 105 & 105 & 106 & 109 & 109 & \\
\hline
\end{tabular}

Zum Beispiel gab es keinen statistisch gesicherten Unterschied bezüglich der berechneten Körperkapazitäten zwischen den Typen „gross-schmal" und „klein-breit", somit 
fand sozusagen ein „Ausgleichprozess" in der Körperkapazität der Typen „grossschmal" und „klein-breit" statt. Dieser „Ausgleichprozess" konnte bei den durch Exterieurbeurteilug geschätzten Körperkapazitäten nicht nachgewiesen werden.

Betrachtet man die Übereinstimmung zwischen den aufgrund der mit beiden Methoden aufgenommenen Daten gebildeten sechs-sechs Typusgruppen, so ergaben sich folgende prozentuale Übereinstimmungen zwischen den Typusgruppen: Die Tiere in den mit den zwei Methoden gebildeten Gruppen waren in den Typusgruppen „kleinschmal" zu $43 \%$, „klein-breit" zu $31 \%$, ,mittelgross-schmal" zu 16\%, ,mittelgrossbreit" zu $32 \%$, ,gross-schmal" zu $32 \%$ und ,gross-breit" zu $35 \%$ identisch. Es kann festgestellt werden, dass der Beurteiler die „kleinen-dünnen" und die „grossen-breiten" Tiere von den anderen etwas effizienter (von zehn Urteilen vier richtig) unterscheiden konnte. Das Ergebnis war bei den vier mittleren Typen noch weniger überzeugend (von zehn Urteilen drei richtig),

Wenn der gesamte Versuchstierbestand in die Gruppen „klein", „mittelgross" und ,gross" eingeteilt wurde, betrug die Übereinstimmung 41, 46 und $54 \%$. Wurde dieser Bestand bezüglich Sitzbeinbreite nur in zwei Gruppen, ,schmal" und „breit" eingeteilt, erreichte die Übereinstimmung 55 bzw. $59 \%$.

Die obigen prozentualen Werte bestätigten, dass erwartungsgemäß die Verringerung der Merkmale und der Kategorien innerhalb der Merkmale, die Zuverlässigkeit der subjektiven Beurteilung verbessert.

Aus den dargestellten Ergebnissen ging hervor, dass es beim Vergleich der Messung und der Beurteilung der Körpermaße nicht ausreicht nur die Mittelwerte der Gruppen / Typusgruppen zu vergleichen. Dieser Vergleich kann zwar, wie in diesem Beispiel gezeigt, scheinbar aussagefähige Ergebnisse bringen. Die Untersuchung der Standardabweichungen und die Regressionsberechnungen - ergänzt mit einer individuellen Registrierung der Tiere - dagegen zeigten, dass die Exterieurbeurteilung mit dem „50Punkte-Beurteilungssystem" subjektiv und unzuverlässig ist, obwohl die Exterieurbeurteilungen durch einen außerordentlich erfahrenen und bewährten Experten durchgefuihrt wurden.

Seit langem wurde angenommen, dass die Beurteiler die Möglichkeiten der breiten Punkteskala (50 Punkte) nicht ausschöpfen. Diese Annahme konnte am Beispiel der untersuchten vier Merkmale eindeutig bestătigt werden. Das neue "9-Punkte-Exterieurbeurteilungssystem " wird zuverlassiger, handlicher und benutzerfreundlicher werden ais das ,,50-Punkte-System", da die Punkteskala weniger Kategorien enthält. Auch das „9-Punkte System" wird nur dann funktionieren, wenn Noten auch in den Extrembereichen der Skala gegeben werden. Die Varianz der Beurteilungspunkte muss also erhöht werden. Es ist auch nicht unwichtig, dass die Punkte weitgehend die Realität widerspiegeln. BREM (1998) empfiehlt daher zentrale Klassifiziererschulungen auf nationaler und internationaler Ebene.

Aufgrund des Vergleichs der zwei Methoden konnte festgestellt werden, dass die durch Exterieurbeurteilung geschätzten Körpermaße und Körperkapazităten den tatsächlichen Körpermassen und Körperkapazitäten der Kühe nur bedingt entsprechen. Es wäre sinnvoll, die Widerristhöhe und die Körpermaße, welche die Körperkapazität beeinflussen (Rumpftiefe, Körperlänge, Brust- und Sitzbeinbreite) im Rahmen der Exterieurbeurteilung zu messen, was mit vertretbarem Aufwand möglich ist. Denn die gemessenen Körpermaße und die berechnete Körperkapazität liefern eine reale Basis 
zum Aufzeigen der Unterschiede zwischen den Typusgruppen. Die berechnete Körperkapazität sollte in Ungarn zu den Selektionskriterien gehören, damit eine Steigerung der bisher vernachlässigten Milchproduktionseffizienz möglich wird. Die Bedeutung der berechneten Körperkapazität wurde in früheren Arbeiten (PÜSKI et al., 1998, 1999) bereits betont. Im Zusammenhang mit steigenden Leistungsforderungen (BOZO, 1992), auch in Bezug auf Nutzungsdauer (GASPARDY et al., 1995) und Langlebigkeit, gewinnen Beziehungen und Abhängigkeiten zu Merkmalen des Exterieurs beim Milchrind und damit deren möglichst exakte Bewertung zunehmend an Bedeutung und fehlen in keinem modernen Zuchtprogramm.

\section{Literatur}

BODROGI,K; DOMA, P.; SOMOS, Z; JOO, L.; PÓCZ, J.:

A magyar holstein-friz küllemi birálata (Exterieurbeurteilung beim ungarischen Holstein-Friesian). A Holstein-friz Tenyésztök Egyesilletének kiadványa (1) Mitteilung des Holstein-Friesian Zuchtvereins, BOZÓ, S.: Ungarn (1)) (1994)

A tenyészcél meghatározását és a szelekció eredményességét elösegitő tényezôk a tejelö szarvasmarha tenyésztésében (Parameter zur Bestimmung des Zuchtziels und zur Steigerung der Effizienz in der Zuchtung des Milchviehs). Kandidátusi értekezés, MTA [Dissertation, Akademie der Wissenschaften, BREM, G.: Ungam] (1992)

GÁSPARDY, A.; BOZÓ, S.; SZÜCS, E.; TRAN, A.T.:

Exterieurbeurteilung landwirtschaftlicher Nutztiere, Verlag Eugen Ulmer, Stuttgart, 1998, 317 pp.

A kullemi tulajdonságok összefuggése a hasznos élettartammal eltéró mamagasságú holstein-friz tehenekben (Zusammenhang von Exterieurmerkmalen und Nutzungsdauer bei Holstein-Friesian Kahen). Állattenyésztés és Takarmányozás, Herceghalom, 44 (1995), 227-241

LEUTHOLD, G.; REINECKE, P.:

Zuchtung auf Futteraufnahmevermoggen. Moglichkeiten und Probleme. Tierzucht, Berlin, 4I (1987), 228-230

PÜSKI, J.; GÁSPÁRDY, A,; TRAN, A.T.; BOZÓ, S.; SZÜCS, E.:

The effect of type on efficiency in Holstein cows. USA, Joint Meeting Abstracts: J. Anim. Sci. Vol. 76, Suppl. 1 / J. Dairy Sci., Vol. 81, Suppl. 1 (1998), 259 p.

PÜSKI, J,; TRAN, A.T.; GÁSPARDY, A.; BOZÓ, S; SZÜCS, E.:

A tipus hatása a holstein tehenek tejtermelésének hatékonyságára az elsó laktációban (Wirkung des Typus auf die Effizienz der Milchproduktion von Holstein-Friesian Kuhen in der ersten Laktation). Allattenyésztés és Takarmányozás, Herceghalom 48 (1999), 323-337

SCHWARK, H.J.; FAHR, R.-D.:

Phänotypische und genetische Analyse der Korperformmerkmale von Jungkuhen des Schwarzbunten Milchrindes. Arch. Tierz., Berlin 32 (1989), 203-213

TRIMMBERGER, G.W.; ETGEN, W, M; GALTON, D. M.: ZELFEL, S: Dairy cattle judging techniques, prentice hall inc. Englewood-Cloffe. New Jersey (1987)

Zur optimalen Lebendmasse unserer Milchkuhe. Tierzucht, Berlin 38 (1984) 10, 469-470

Eingegangen: 14.03 .2000

Akzepticrt: 27.11 .2000

Anschriften der Verfasser

Dr. JANOS PUSKI, Prof. Dr. ENDRE SZÚCS

Szent István Universität

Fakultăt für Agrarwissenschaften

H-2103 Gődőllŏ, Ungarn

Dr, SÁNDOR BOZÓ, PhD

Dr. ISTVÁN GYÖRKÖS

Institut fiur Tierzucht und Tierernăhrung

Herceghalom, Ungam

Dr. ANDRÁS GÁSPÁRDY

Szent István Universităt, Fakultăt für Veterinärmedizin

Budapest, Ungarn 
Arch: Tierz, Dummerstorf 44 (2001) 1, 46

\title{
Buchbesprechung
}

\author{
Handbuch zur Pflege und Behandlung der Klauen beim Rind \\ CHRISTOPH LISCHER (Hrsg.), PETE OSSENT, KATHRIN FRIEDLI, IMMANUEL NÄF, RENE PIJL.
}

158 Seiten, 184 Farbfotos, 70 farbige Zeichnungen, Parey Buchverlag, Berlin, 2000, ISBN 3-8263-3331-4, DM 49,$80 ; 8$ S 364,00, sFr 46,00

Gesunde Klauen sind nicht nur, aber besonders für Hochleistungskuhe eine wichtige Voraussetzung für Gesundheit und eine dauerhafte Leistung. Das triff um so mehr fur Tiere zu, die uberwiegend in Stallungen ohne ausreichende Bewegung auf Spaltenböden gehalten werden. Eine ständige Überwachung der Klauen und die Erhaltung oder Wiederherstellung der natülichen Funktionen durch eine sachgemaßße Klauenpflege sind daher für eine erfolgreiche Rinderhaltung unabdingbar. Eine fachgerechte Durchfulhrung ist abhăngig von einer guten Ausbildung und einem fundierte Wissen und dieses soll das vorliegende Buch vermitteln. Gleichzeitig soll es auch den Tierhalter besser befahigen Probleme und Krankheiten an den Klauen zu erkennen und die Zusammenarbeit mit dem Klauenpfleger oder dem Tierarzt effektiver zu gestalten.

Besonders zu erwăhnen ist die hervorragende Farbfotoausstattung des Buches sowie die anschaulichen, die Textaussagen wirkungsvoll unterstutzenden, ausgezeichneten farbigen Zeichnungen.

Das Buch ist in 11 Hauptabschnitte untergliedert. Nach dem Abschnitt der Anatomie der Rinderklaue, folgt die Biomechanik und die Beschreibung der Hilfsmittel für eine erfolgreiche Klauenpflege. Sehr praxisrelevant werden anschließend die Technik der Klauenpflege in ihrer Abfolge und unter Nutzung verschiedener Hilfsmittel, die hăufigsten Fehler bei der Ausführung sowie, als besonderem Abschnitt, in Wort und Bild die funf Arbeitsschritte der sogenannten ,funktionellen Klauenpflege" beschrieben. Die nachfolgenden Beiträge widmen sich der Behandlung häufiger Klauenkrankheiten, ihrer Beschreibung, den Ursachen und vorbeugenden Maßnahmen zur Verhutung von Klauenproblemen. Die beiden letzten Abschnitte beschäftigen sich mit dem Baden der Klauen zur Desinfektion und den hierbei angewandten Mitteln der Wahl sowic der Untersuchungen der Klauen nach der Schlachtung.

Dieses selur praxisrelevante Buch hălt, was es verspricht. Schwerpunkte sind die Anatomie, praktische Klauenpflege und deren Hilfsmittel, die Beschreibung der hăufigsten Klauenkrankheiten, ihre Vorbeuge und Behandlung. Diese Reihenfolge könnte man sich auch für die Buchgliederung vorstellen. Hilfreich werden die gut verstandlichen Aussagen und Empfehlungen durch die farbigen Textzeilen unterstützt, die in allen Abschnitten auf beachtenswerte Besonderheiten hinweisen. Dieses Buch ist allen in der praktischen Rinderhaltung Tătigen, den Auszubildenden, den Klauenpflegern, Tierärzten und Fachberatern zu empfehlen. 